Research Paper

\title{
P-Selectin-Mediated Adhesion between Platelets and Tumor Cells Promotes Intestinal Tumorigenesis in $\mathrm{Apc}^{\mathrm{Min} /+}$ Mice
}

\author{
Cuiling $\mathrm{Qi}^{1}{ }^{1}{ }^{*}$, Bin $\mathrm{Li}^{2 *}$, Simei Guo ${ }^{2}$, Bo Wei ${ }^{3}$, Chunkui Shao ${ }^{3}$, Jialin $\mathrm{Li}^{2}$, Yang Yang ${ }^{2}$, Qianqian Zhang ${ }^{2}$, \\ Jiangchao $\mathrm{Li}^{2}$, Xiaodong $\mathrm{He}^{2}$, Lijing Wang ${ }^{2}$, Yajie Zhang ${ }^{\circledR}$ \\ 1. Department of Pathology, Guangzhou Medical University, Guangzhou, Guangdong 510182, China \\ 2. Vascular Biology Research Institute, Guangdong Pharmaceutical University, Guangzhou, Guangdong 510006, China \\ 3. Department of Gastrointestinal Surgery and Department of Pathology, the Third Affiliated Hospital, Sun Yat-sen University, Guang- \\ zhou, Guangdong 510630, China \\ * These authors contributed equally to this work.
}

$\triangle$ Corresponding authors: Yajie Zhang or Lijing Wang. Address: Department of Pathology, Guangzhou Medical University, 195 Dongfengxi Road, Guangzhou, 5101 82, P.R. China, or Vascular Biology Research Institute, Guangdong Pharmaceutical University, 280 Waihuandong Road, Guangzhou, 510006, P.R. China; Tel: +86-13922253597; E-mail: Yajie.Zhang@163.com or wanglijing62@163.com

(C) 2015 Ivyspring International Publisher. Reproduction is permitted for personal, noncommercial use, provided that the article is in whole, unmodified, and properly cited. See http://ivyspring.com/terms for terms and conditions.

Received: 2015.01.15; Accepted: 2015.03.24; Published: 2015.04.29

\begin{abstract}
Studies have indicated that platelets play an important role in tumorigenesis, and an abundance of platelets accumulate in the ovarian tumor microenvironment outside the vasculature. However, whether cancer cells recruit platelets within intestinal tumors and how they signal adherent platelets to enter intestinal tumor tissues remain unknown. Here, we unexpectedly found that large numbers of platelets were deposited within human colorectal tumor specimens using immunohistochemical staining, and these platelets were fully associated with tumor development. We further report the robust adhesion of platelet aggregates to tumor cells within intestinal tumors, which occurs via a mechanism that is dependent on P-selectin (CD62P), a cell adhesion molecule that is abundantly expressed on activated platelets. Using spontaneous intestinal tumor mouse models, we determined that the genetic deletion of P-selectin suppressed intestinal tumor growth, which was rescued by the infusion of wild-type platelets but not P-selectin ${ }^{-1-}$ platelets. Mechanistically, platelet adhesion to tumor cells induced the secretion of vascular endothelial growth factor (VEGF) to promote angiogenesis and accelerate intestinal tumor cell proliferation. Our results indicate that the adherence of platelets to tumor cells could promote tumor growth and metastasis. By targeting this platelet-tumor cell interaction, recombinant soluble P-selectin may have therapeutic value for the treatment of intestinal tumors.
\end{abstract}

Key words: P-selectin; platelets; intestinal tumor; VEGF

\section{Introduction}

It is well known that platelets play key roles in tumor growth and metastasis. The clinical observation was made that patients with solid tumors, most commonly primary colorectal, lung, breast or gastrointestinal tumors, often have thrombocytosis [1]. A recent report also showed that increased platelet counts promote ovarian tumor growth via a paracrine signaling pathway that mediates paraneoplastic thrombocytosis in epithelial ovarian tumors [2]. Experimental evidence also showed that platelets exert important effects on tumorigenesis through diverse mechanisms, including the release of several types of 
growth factors, protection of cancer cells from immune surveillance, and negotiation of cancer-cell arrest in the micro-vasculature [3-5]. Platelets can induce cell survival and growth by secreting several types of growth factors, including vascular endothelial growth factors (VEGFs), platelet-derived growth factors (PDGFs) and fibroblast growth factors (FGFs). Although it has been confirmed that platelets can promote tumorigenesis [6,7], the application of platelet depletion is clinically limited due to potentially fatal hemorrhage. Thus, when treating cancers, it is highly necessary to block interactions between platelets and tumor cells in solid tumors without causing bleeding.

P-selectin (CD62P) is a member of the selectin family of cell adhesion molecules and is stored on the membrane of platelet $\alpha$-granules and endothelial Weibel-Palade bodies [8]. When thrombosis develops and inflammation is caused, P-selectin rapidly transfers to the cell surfaces of platelets and endothelial cells. Platelet P-selectin mediates leukocyte rolling on stimulated endothelial cells via binding to PSGL-1 and the heterotypic aggregation of activated platelets with circulating tumor cells (CTCs) [9]. It has also been shown that the absence of P-selectin significantly suppresses the growth of subcutaneously implanted human colon carcinoma cells and lung metastases from intravenously injected cells [10]. Indeed, P-selectin has been suggested to play an important role in tumor growth and metastasis, and the pathologic significance of P-selectin-mediated platelet binding to CTCs during hematogenous metastasis has been clearly demonstrated [9-12]. However, whether, why and how P-selectin-mediated platelets adhere to tumor cells within solid tumors currently remain unknown.

To investigate platelet $\mathrm{P}$-selectin involved in intestinal tumors, the $\mathrm{Apc}^{\mathrm{Min} /+}$ murine model is used. The Apc $\mathrm{Ain}^{\mathrm{+}}$ mouse is an intestinal tumor model that carries a mutation at codon 850 of the human adenomatous polyposis coli (Apc) gene [13]. Apc ${ }^{\mathrm{Min} /+}$ mice spontaneously develop multiple adenomatous polyps in the small intestine and fewer polyps in the colon, as a consequence of the mutation in the Apc gene in multiple intestinal neoplasia (Min). Very few tumors in $\mathrm{Apc}^{\mathrm{Min} /+}$ mice develop into adenocarcinoma [13]. Therefore, $\mathrm{Apc}^{\mathrm{Min} /+}$ mice have been considered a classic mouse model to study human intestinal tumorigenesis.

In this study, we investigated whether platelet P-selectin plays an important role in tumorigenesis by monitoring $\mathrm{Apc}^{\mathrm{Min} /+}$ intestinal tumorigenesis in a P-selectin-deficient background. Surprisingly, we found that platelet deposition within colorectal cancers was closely correlated with tumor staging and lymph node metastasis, demonstrating that platelet accumulation in tumor tissue promoted colorectal cancer growth. We further demonstrated that P-selectin deletion inhibited tumor growth in both adenoma and microadenoma and attenuated platelet deposition within adenomas in $\mathrm{Apc}^{\mathrm{Min} /+}$ mice, thus reducing the secretion of VEGF and suppressing tumor-induced angiogenesis, which ultimately inhibited intestinal tumor growth.

Although decreasing platelets is not feasible in clinical applications due to potential hemorrhage [6,7], a novel method to treat colorectal cancer could involve targeting the newly identified platelet-tumor cell interaction. In this context, P-selectin was found to be an effective candidate. The current study aimed to confirm the role of P-selectin-mediated platelet deposition in intestinal tumor growth and to provide a strong theoretical basis for P-selectin inhibition as an anti-tumor treatment.

\section{Materials and Methods}

\section{Human samples}

Human tissues were obtained from patients undergoing elective surgery for colorectal resections with written informed consent after approval from the Medical Research Human Ethics Committee of the Third Affiliated Hospital of Sun Yat-sen University. For immunofluorescent or immunohistochemical staining, the tissues were fixed in $4 \%$ paraformaldehyde and embedded in optimum cutting temperature (OCT) compound or in paraffin. The paraffin-embedded tissues included adenoma tissues $(\mathrm{n}=$ 3 ) and colorectal cancer tissues at stage I $(n=4)$, II ( $=10)$, III $(\mathrm{n}=10)$, and IV $(\mathrm{n}=4)$; the tissues were chosen according to the original pathological diagnosis. Hematoxylin and eosin (H\&E)-stained sections were re-diagnosed by two experienced pathologists who were blinded to the source of the tissues, according to the American Joint Committee on Cancer Staging (AJCC) and Union for International Cancer Control (UICC) classification guidelines.

\section{Mice}

C57BL/6J-Apc ${ }^{\mathrm{Min} /+}$ mice were purchased from the Jackson Laboratory. Male $\mathrm{Apc}^{\mathrm{Min} /+}$ mice were mated with female $\mathrm{P}$-sel $/-$ mice on the C57BL/6J background to obtain $\mathrm{Apc}^{\mathrm{Min} /+} ; \mathrm{P}_{-} \mathrm{sel}^{+/-}$mice. To generate $\mathrm{Apc}^{\mathrm{Min} /+} ; \mathrm{P}-\mathrm{sel}^{-/-}$mice, the $\mathrm{Apc}^{\mathrm{Min} /+} ; \mathrm{P}_{-} \mathrm{sel}^{+/-}$mice were backcrossed with $\mathrm{P}$-sel-/- mice. Their genotypes were identified by PCR using the following primers: Apc $^{\mathrm{Min} /+}$, GCCATCCCTTCACGTTAG, TTCCACTTT GGCATAAGGC and TTCTGAGAAAGACAGAAG TTA; and P-selectin, CTGAATGAACTGCAGG ACGA, ATACTTTCTCGGCAGGAGCA, TTGTAAAT CAGAAGGAAGTGG and AGAGTTACTCTTGAT 
GTAGATTCC.

\section{Measurement of intestinal polyps}

After the mice were sacrificed, the intestines were excised from each mouse. To easily quantify and identify tumors, the small intestine was divided into three equal segments: proximal, middle and distal. The intestines were cut longitudinally and luminal contents were washed out using phosphate buffered saline solution (PBS, $\mathrm{pH} 7.4$ ). After unfolding on filter papers, the intestines were fixed in $10 \%$ phosphate-buffered formalin overnight. The next day, the intestines were stained with $0.1 \%(\mathrm{v} / \mathrm{v})$ methylene blue. The numbers and diameters of the tumors in the small and large intestines were measured using an inverted microscope, and tumor volumes were calculated with the following equation: volume $=0.52 \times$ (length $\mathrm{x}$ width ${ }^{2}$ ) [14]. Based on tumor diameters, the tumors were categorized as microadenoma $(<2 \mathrm{~mm})$ and adenoma ( $\geq 2 \mathrm{~mm}$ ). The intestines were prepared in a Swiss roll and embedded in paraffin.

\section{Platelet preparation and "rescue" experi- ments}

Blood from P-sel-/- or wild-type mice was collected in Eppendorf tubes containing 0.1 volume of acid-citrate-dextrose (ACD, $38 \mathrm{mmol} / \mathrm{L}$ citric acid, 75 $\mathrm{mmol} / \mathrm{L}$ trisodium citrate, and $100 \mathrm{mmol} / \mathrm{L}$ dextrose, $1 / 10$ vol). To obtain platelet-rich plasma (PRP), blood was centrifuged at $275 \mathrm{~g}$ for 14 minutes, and then the plasma and buffy coat composed of platelets were gently drawn into a fresh tube and centrifuged at 400 $\mathrm{g}$ for 5 minutes. The resulting PRP was filtered to obtain the platelets using a Sepharose 2B column (Sigma-Aldrich, St. Louis, MO, USA) containing PIPES buffer (25 mmol/L PIPES, $137 \mathrm{mmol} / \mathrm{L} \mathrm{NaCl}, 4$ $\mathrm{mmol} / \mathrm{L} \mathrm{KCl}$, and $0.1 \%$ dextrose, $\mathrm{pH} 7.0$ ). Counting of platelets was performed using a blood cell counting chamber in a $1000 \times$ field. For the platelet "rescue" experiments, platelets $\left(4 \times 10^{7} /\right.$ mouse $)$ isolated from $\mathrm{C} 57 \mathrm{BL} / 6 \mathrm{~J}$ and $\mathrm{P}_{-} \mathrm{sel}^{-/-}$mice were intravenously injected into $\mathrm{Apc}^{\mathrm{Min} /+} ; \mathrm{P}-\mathrm{sel}^{-/-}$mice (5 weeks old; three times per week for four weeks). Four weeks later, the tumor numbers and volumes of the 9-week-old $\mathrm{Apc}^{\mathrm{Min} /+}, \mathrm{P}-\mathrm{sel}^{-/-}$mice were evaluated as described in the previous methods section.

\section{Histopathology and immunohistochemical staining}

Paraffin-embedded intestines were sectioned and stained with H\&E according to a standard protocol. Two senior pathologists graded the stained slides and defined the adenomas as low or high grade according to the consensus recommendations [15]. Before immunohistochemical staining, 5-bromo- 2'-deoxyuridine (BrdU, $100 \mathrm{mg} / \mathrm{kg}$; Sigma-Aldrich) was intraperitoneally injected into 9-week-old $\mathrm{Apc}^{\mathrm{Min} /+}, \mathrm{P}-\mathrm{sel}^{-/-}$or $\mathrm{Apc}^{\mathrm{Min} /+}$ mice, and the mice were sacrificed after $2 \mathrm{~h}$. Three-micron tissue sections were subsequently dewaxed, deparaffinized, rehydrated, and endogenous peroxidase was quenched with $3 \%$ $\mathrm{H}_{2} \mathrm{O}_{2}$ in methanol for 30 minutes. The slides were blocked with $10 \%$ bovine serum albumin (Sigma-Aldrich) at $37^{\circ} \mathrm{C}$ for 50 minutes and then incubated with a rabbit anti-CD31 polyclonal antibody (Abcam, Cambridge, CB, UK), mouse anti-BrdU monoclonal antibody (Santa Cruz Biotechnology, Santa Cruz, CA, USA), rabbit anti-CD41 (integrin GPIIb subunit) polyclonal antibody (Abcam) or rabbit anti-VEGF polyclonal antibody (Abcam) at $4^{\circ} \mathrm{C}$ overnight. Next, the tissue sections were incubated with a horseradish peroxidase-conjugated goat anti-rabbit or anti-mouse IgG antibody (ZSGB-BIO, Beijing, China) at $37^{\circ} \mathrm{C}$ for 50 minutes. The slides were stained with diaminobenzidine solution (Dako Cytomation, Hamburg, Germany), and the cell nuclei were counterstained with hematoxylin. The images were taken under a 200 $\times$ field or $400 \times$ field. Microvessel density (MVD) and the numbers of proliferating cells were quantified according to previously published methods [16]. The data of immunohistochemical staining were collected and assessed by two reseachers using a double-blind protocol.

\section{Immunofluorescent staining}

Immunofluorescent staining was performed using antibodies against CD31 (Santa Cruz Biotechnology) and CD41 (Abcam), and cell nuclei were counterstained with 4'-6-diamidino-2-phenylindole (DAPI). Image-Pro Plus image analysis software was used to quantify the platelets under a $400 \times$ field, and images were assessed by two researchers.

\section{ELISA}

The tumors obtained from $\mathrm{Apc}^{\mathrm{Min} /+}$ mice and $\mathrm{Apc}^{\mathrm{Min} /+}, \mathrm{P}-\mathrm{sel}^{-/-}$mice were homogenized. The homogenates, after being subjected to two freeze-thaw cycles, were used to detect VEGF levels using an ELISA kit (R\&D Systems, Minneapolis, MN, USA) according to the protocol provided by the manufacturer (R\&D Systems).

\section{Western blotting}

Protein was extracted from the adenomas obtained from $\mathrm{Apc}^{\mathrm{Min} /+}$ mice and $\mathrm{Apc}^{\mathrm{Min} /+} ; \mathrm{P}-\mathrm{sel}^{-/-}$mice, and then the proteins were separated by SDS-PAGE and transferred onto nitrocellulose membranes. The nitrocellulose membranes were incubated with a rabbit anti-VEGF polyclonal antibody (Abcam) at $4^{\circ} \mathrm{C}$ overnight, and the immunoblotting was carried out as previously described [14]. 


\section{Statistical analysis}

Data were represented as the mean \pm standard deviation (SD). A one-way ANOVA or Student's $t$-test was used to determine the statistical significance. For all tests, $p<0.05$ or $<0.01$ indicated statistical significance and high significance, respectively.

\section{Results}

\section{Clinical evidence for platelet infiltration in human colorectal tumors}

It has been reported that decreasing the number of platelets in host blood inhibits tumor growth and metastasis [17]. To confirm whether platelets could infiltrate into colorectal tumor tissues from blood vessels and to evaluate the relationship between platelets and tumorigenesis, we detected platelet accumulation in human colorectal specimens using immunohistochemical staining. Interestingly, we found that many platelets aggregated around colorectal tumor cells. In particular, there were large numbers of platelets in colorectal cancer tissues from stage III and IV patients (Figure 1A). Furthermore, statistical analysis indicated that platelet accumulation in stage II tumors was greater than in stage I, and stage IV was more than stage III; furthermore, there was no difference in platelet accumulation between adenoma and stage $0 / \mathrm{I}$ and between stage $0 / \mathrm{I}$ and stage II (Figure 1B). Surprisingly, we found that platelet aggregates were closely correlated with lymph node metastasis, and platelet accumulation in stage T4 colorectal cancer was greater than in stage T3 tumors (Figures 1C and 1D). However, there was not platelet deposition in the normal intestinal tissues (Supplemental Figure S1). The results indicate that platelet accumulation around tumor cells is closely related to colorectal cancer progression.

\section{P-selectin mediates platelet accumulation in the intestinal tumor}

P-selectin is expressed on activated platelets and endothelial cells and has an important effect on the adhesion of tumor cells to vascular endothelial cells. Therefore, to determine why platelets accumulate in human colorectal tumor tissues and why platelets accumulation is closely related to tumor progression (Figure 1), we established $\mathrm{Apc}^{\mathrm{Min} /+} ; \mathrm{P}-\mathrm{sel}^{-/-}$mice and further detected platelets in the small intestinal tumors of $\mathrm{Apc}^{\mathrm{Min} /+} ; \mathrm{P}-\mathrm{sel}^{-/}$and $\mathrm{Apc}^{\mathrm{Min} /+}$ mice using immunofluorescent staining of CD41. In addition to platelets accumulation within blood vessels, we found the large number of platelets accumulates within the small intestinal tumor of the Apc $\mathrm{Min} /+^{+}$mice. However, few platelets were detected in the small intestinal tumor of $\mathrm{Apc}^{\mathrm{Min} /+} ; \mathrm{P}-\mathrm{sel} /-$ mice (Figure 2). These results show that P-selectin mediates platelet accumulation in intestinal tumor.

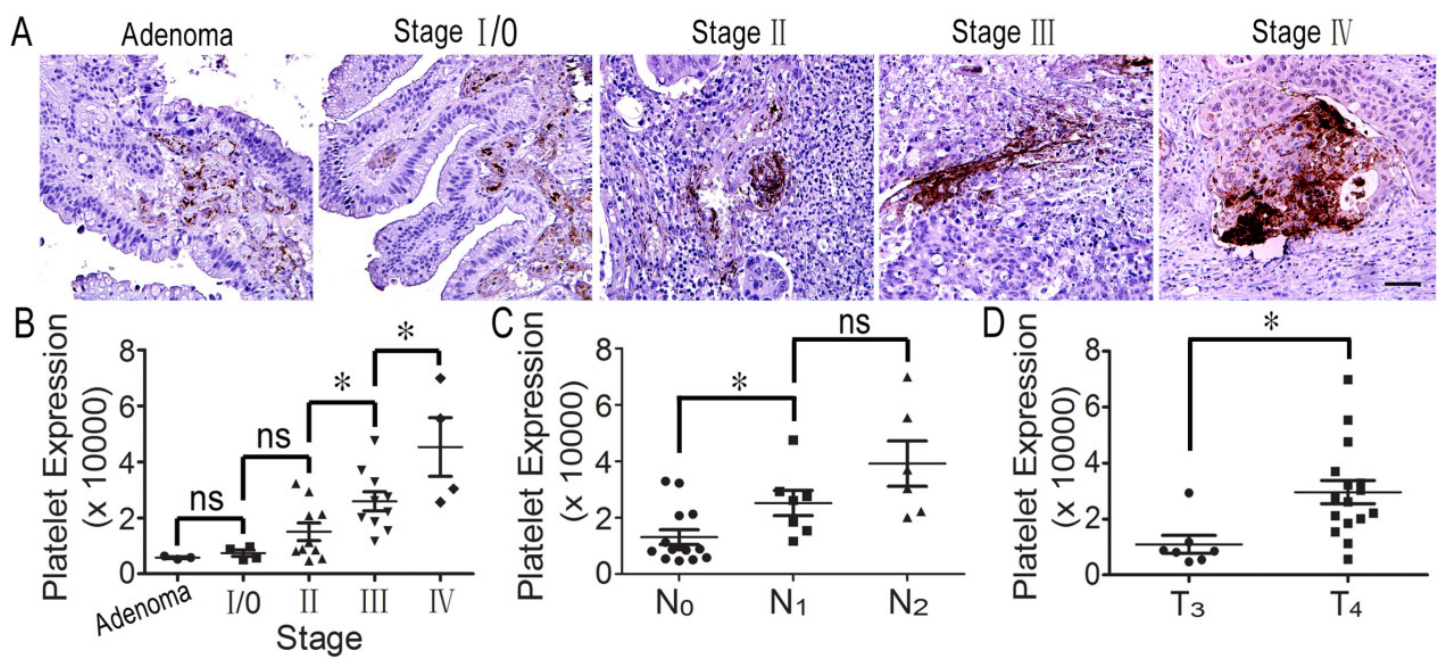

Figure 1. Platelets accumulate in human colorectal tumors. (A) Platelets deposited in human specimens at serious colorectal tumor stages. (B) Platelet expression across adenoma and individual AJCC stages (I-IV). There was no difference in platelet expression between adenoma and stage 0/I. Platelet expression was significantly increased in stage III colorectal cancer compared with stage II. There were greater numbers of platelets in stage IV cancer than in stage III tumors. (C) Platelet expression in colorectal cancers with no lymph node metastasis $\left(\mathrm{N}_{0}\right)$ was significantly decreased compared to those in the tissues with metastasis in $1-3$ lymph nodes $\left(\mathrm{N}_{1}\right)$ and metastasis in more than 4 lymph nodes $\left(\mathrm{N}_{2}\right)$. (D) Platelet accumulation in the stage IV colorectal cancer group was significantly increased compared to stage III. $*, p<0.05$; ns, not significant. Scale bars $=50 \mu \mathrm{m}$. 


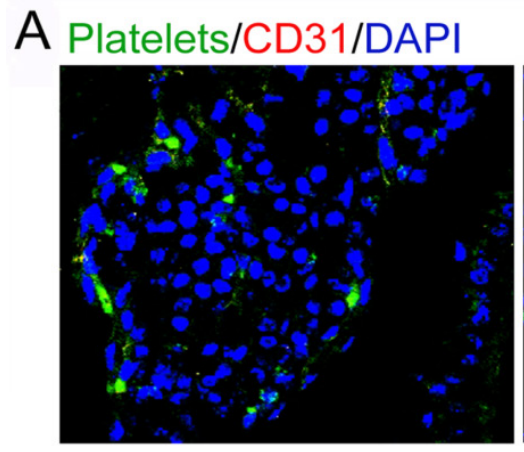

$\mathrm{Apc}^{\mathrm{Min} /+}$

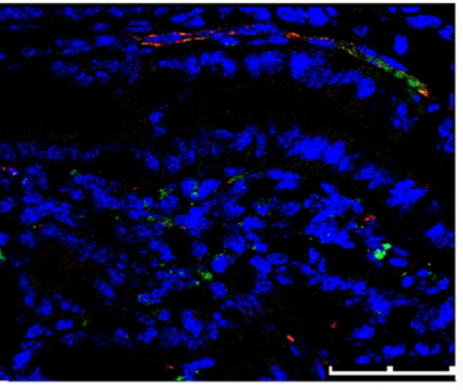

$\mathrm{Apc}^{\mathrm{Min} /+} ; \mathrm{P}-\mathrm{sel}^{-/-}$

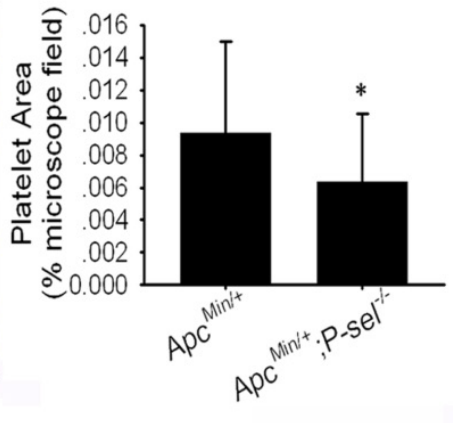

Figure 2. P-selectin mediated platelet accumulation in intestinal tumors. Immunofluorescent staining was performed with an anti-CD31 antibody to define blood vessels, a $\beta \mathrm{Ilb}$ antibody for platelets and counterstaining of nuclei with DAPI. Immunofluorescent images of small intestine adenomas from ApcMin/+ and $\mathrm{Apc}^{\mathrm{Min} /+} ; \mathrm{P}-$ sel $^{-/-}$mice were taken. There were more platelets in the adenomas from Apc ${ }^{\mathrm{Min} /+}$ mice than from Apc ${ }^{\mathrm{Min} /+} ; \mathrm{P}-\mathrm{sel}^{-/-} \mathrm{mice}^{*} *, p<0.05$. Scale bars $=50 \mu \mathrm{m}$.

\section{P-selectin-mediated platelet accumulation accelerates intestinal tumor growth}

To determine the role of P-selectin-mediated platelet in tumor growth in $\mathrm{Apc}^{\mathrm{Min} /+}$ mice, we further evaluated the pathological significance of P-selectin deletion in intestinal tumor. Most tumors were found in the small intestines, and the number of both the microadenoma and adenoma in the small intestines and the number of total tumors were significantly reduced in $\mathrm{Apc}^{\mathrm{Min} /+} ; \mathrm{P}_{-}-\mathrm{sel}^{-/-}$mice compared to $\mathrm{Apc}^{\mathrm{Min} /+}$ mice (Figures $3 \mathrm{~A}$ and $3 \mathrm{~B}$ ). P-selectin deficiency also reduced tumor volume of both microadenoma and adenoma in the small and the volume of total tumors (Figure 3C). These results indicate that P-selectin deletion plays an important role in intestinal tumor promotion. Both low- and high-grade adenomas (Figures 3D and 3E) were found in $\mathrm{Apc}^{\mathrm{Min} /+}$ mice and low-grade adenomas were more than high-grade adenomas (Figures 3D) [18]. However, high-grade adenomas were very few in $\mathrm{Apc}^{\mathrm{Min} /+} ; \mathrm{P}-\mathrm{sel}^{-/-}$mice. Furthermore, both low-grade and high-grade adenomas in $\mathrm{Apc}^{\mathrm{Min} /{ }^{+} ; \mathrm{P}-\mathrm{sel}^{-/} \text {mice }}$ were much less than those in $\mathrm{Apc}^{\mathrm{Min} /+}$ mice (Figures 3D).

\section{The significance of platelet P-selectin on in- testinal tumor growth}

Not only activated platelets but also vascular endothelial cells express P-selectin [8]. To determine the significance of platelet P-selectin on intestinal tumor growth, the platelets from C57BL/6J and $\mathrm{P}$-sel $/$ - mice were isolated and "rescue" experiment was performed. Just as predicted, compared to those treated with platelets from C57BL/6J mice, $\mathrm{Apc}^{\mathrm{Min} /+} ; \mathrm{P}-\mathrm{sel}^{-/-}$mice treated with $\mathrm{P}-\mathrm{sel}^{-/-}$platelets developed less tumors, and the tumor volumes were smaller (Figures 4A and 4B). Both low- and high-grade adenomas (Figures $4 \mathrm{C}-4 \mathrm{E}$ ) were found in $\mathrm{Apc}^{\mathrm{Min} /+} ; \mathrm{P}-\mathrm{sel}^{-/-}$mice received from C57BL/6J plate- lets and low-grade adenomas were more than high-grade adenomas (Figures 4C-4E). However, in $\mathrm{Apc}^{\mathrm{Min} /+} ; \mathrm{P}-\mathrm{sel}^{-/-}$mice received from $\mathrm{P}-\mathrm{sel}^{-/}$- platelets, high-grade adenomas was very few. Both low- and high-grade adenomas were more in the $\mathrm{Apc}^{\mathrm{Min} /+} ; \mathrm{P}-$ sel $^{-/}$- mice received from C57BL/6J platelets than those in the mice treated with $\mathrm{P}-\mathrm{sel}^{-/}$- platelets (Figures 4C-4E).

\section{Adherent platelets induce angiogenesis through up-regulating VEGF}

To investigate how adherent platelets promote intestinal tumor growth, IHC staining for CD31 and BrdU cell proliferation assay were performed. MVD and the number of BrdU-positive cells were especially counted under a $200 \times$ field or $400 \times$ field using the upright microscope, and the statistical analysis demonstrated that there were fewer microvessels and BrdU-positive cells in the tumor of $\mathrm{Apc}^{\mathrm{Min} /+} ; \mathrm{P}-\mathrm{sel}^{-/-}$ mice than those in the tumors of $\mathrm{Apc}^{\mathrm{Min} /+}$ mice (Figures $5 \mathrm{~A}$ and $5 \mathrm{~B}$ ). As platelets secrete abundant VEGF $[4,5]$, which is a key factor for promoting angiogenesis in intestinal tumors, we detected the expression and concentration of VEGF in the small intestinal tumors of $\mathrm{Apc}^{\mathrm{Min} /+}$ and $\mathrm{Apc}^{\mathrm{Min} /+} ; \mathrm{P}_{-}-\mathrm{sel}^{-/-}$mice using IHC staining, western blotting and ELISA. As expected, VEGF was highly expressed in the tumor of $\mathrm{Apc}^{\mathrm{Min} /+}$ mice (Figures 5C and 5D), and the levels of VEGF in the tissue lysates of intestinal tumors isolated from $\mathrm{Apc}^{\mathrm{Min} /+}$ mice were higher than those from $\mathrm{Apc}^{\mathrm{Min} /+} ; \mathrm{P}-\mathrm{sel}^{-/-}$mice (Figure 5E).

\section{Discussion}

During wound injury, platelets are recruited to the lesion site, where they adhere and aggregate to form clots that prevent excessive bleeding. They also secrete a myriad of growth factors, cytokines and chemokines in situ, which dictate the reparative process of wound healing [12]. In this study, we have 
shown that platelets are actively recruited to human colorectal tumor and mouse intestinal adenoma in a P-selectin-dependent manner, where they secrete VEGF, leading to angiogenesis and tumor growth. Our findings thus provide experimental evidence to support the outstanding and provocative hypothesis that solid tumors may hijack the physiological process of wound healing to their own advantage during tumorigenesis $[6,7,19]$.

In the study, we found that platelets accumulated in human colorectal cancer tissue in a manner that was fully consistent with tumor staging and lymph node metastasis (Figure 1). To explore the mechanism by which platelets infiltrated into human colorectal tumors, Apc ${ }^{\mathrm{Min} /+} ; \mathrm{P}-\mathrm{sel}^{-/-}$mice were established, and P-selectin deficiency attenuated intestinal tumor growth by inhibiting the accumulation of platelets in tumors (Figures 2 and 3). These results show that P-selectin mediates platelet accumulation in intestinal tumors. Rebecca L. et al. reported that thrombocytosis shortens the survival of patients with epithelial ovarian cancer and that there is platelet accumulation in ovarian cancer tissue [2], which is fully consistent with our results regarding platelet deposition in intestinal tumors. Our previous results also showed that P-selectin-mediated platelet/CTC interactions promoted melanoma metastasis [16]. Taken together, our results from two different experimental settings demonstrate that P-selectin deficiency may inhibit intestinal tumor growth, possibly by mediating platelet adhesion to tumor cells.
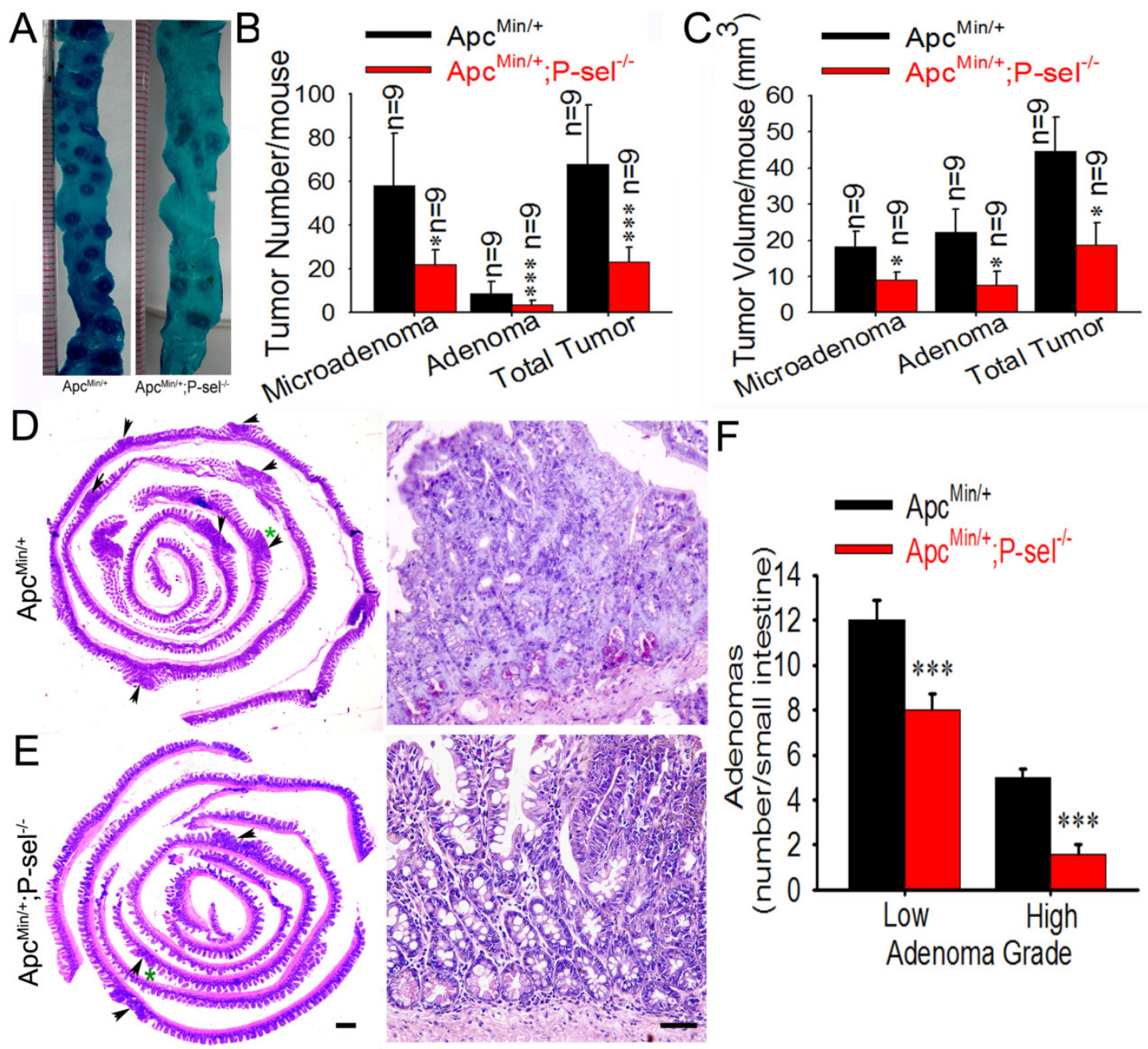

Figure 3. P-selectin-mediated platelet adhesion promotes intestinal tumor growth in ApcMin/+ mice. (A) Representative dissecting gross image of the middle small intestine from ApcMin/+ and ApcMin/+;P-sel-/- mice. (B) The effects of P-selectin deficiency on the numbers of microadenomas, adenomas and total tumors. (C) The effects of P-selectin deletion on the tumor volumes of microadenomas, adenomas and total tumors. (D and E) Representative H\&E stained images. The arrowheads indicate adenomas in the left image. The adenoma indicated by the green asterisk was further magnified in the right image. The adenomas in ApcMin/+ mice and $\mathrm{Apc}$ Min/+;P-sel-/- mice were high-grade and low-grade, respectively. $(\mathrm{F})$ The numbers of low- and high-grade adenomas in the small intestine of ApcMin/+ mice and Apc Min/+;P-sel-/- mice. $*, p<0.05 ; * * *, p<0.001$. Scale bars $=50 \mu \mathrm{m}$ in $\mathrm{E}$. 


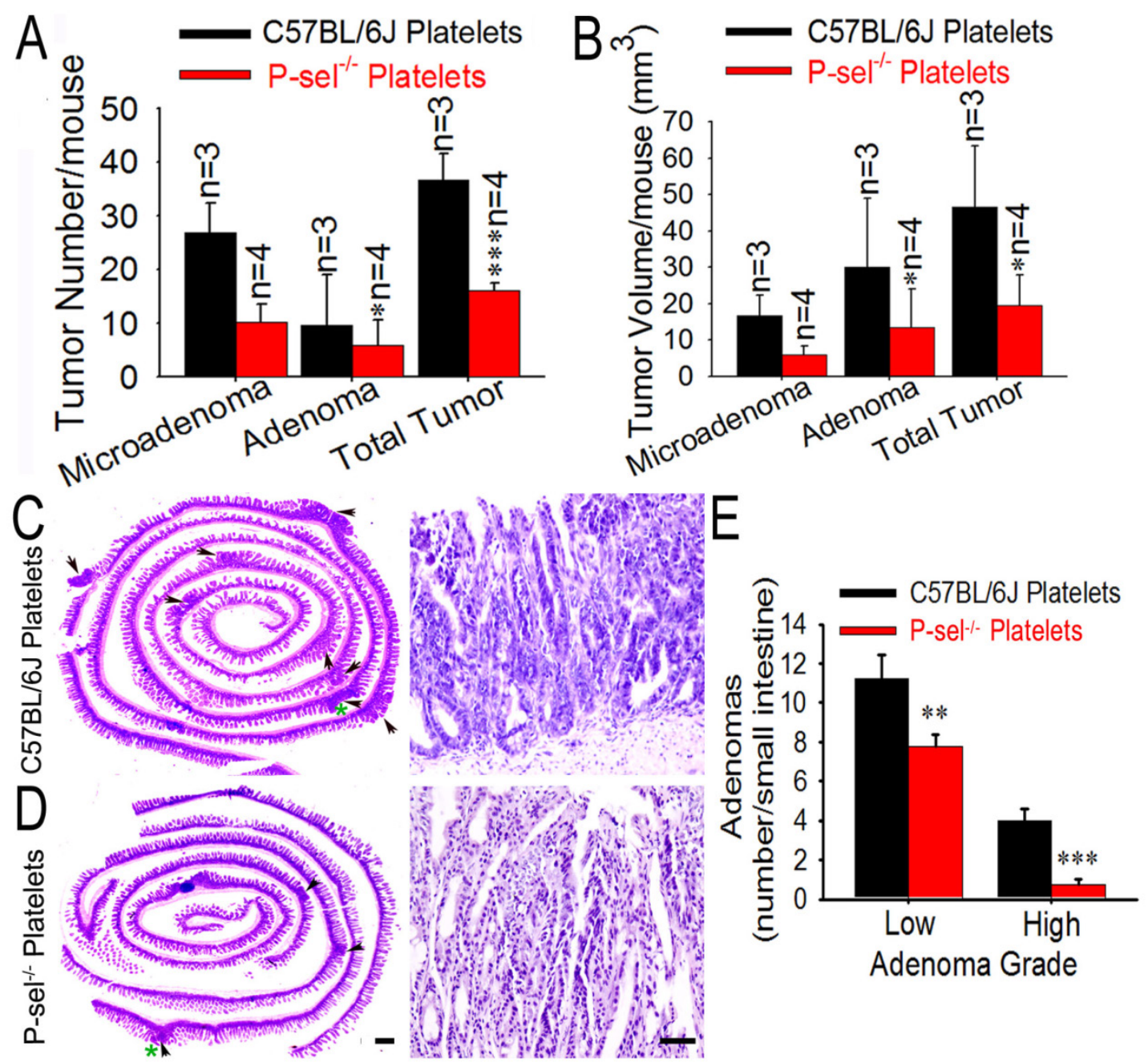

Figure 4. Platelet P-selectin exerts an important role in intestine tumor growth. (A and $B$ ) Influence of platelet $P$-selectin in intestinal tumor growth. After P-sel-/- and wild-type platelets were intravenously injected into ApcMin/+;P-sel-/- mice, tumor number (A) and volume (B) were measured. (C and D) Representative H\&E stained images. The arrowheads indicate adenomas in the left image. The adenoma indicated by the green asterisk was further magnified in the right image. The adenomas in ApcMin/+;P-sel-/- mice administered wild-type and P-sel-/- platelets were high-grade and low-grade, respectively. (E) After platelets from C57BL/6 and $\mathrm{P}$-sel/- mice were intravenously injected into ApcMin/+;P-sel-/- mice, the intestines were stained with $\mathrm{H} \& \mathrm{E}$, and numbers of low- and high-grade adenomas in the small intestine were assessed. *, $p<0.05 ; * *, p<0.01 ; * * *, p<0.001$. Scale bars $=50 \mu \mathrm{m}$.

P-selectin is expressed on activated platelets and stimulated endothelial cells. Although endothelial P-selectin plays an important role in mediating leukocyte rolling on endothelial cells during inflammation [8-10], the physiological and pathological roles of platelet P-selectin in the processes of hemostasis, thrombosis, wound healing and tumorigenesis have not been clearly identified. To explore whether platelet P-selectin has a significant effect on intestinal tumor growth, a platelet "rescue" experiment was performed. We found that platelet P-selectin, but not endothelial cell P-selectin, played a significant role in intestinal tumor growth in $\mathrm{Apc}^{\mathrm{Min} /{ }^{+}} ; \mathrm{P}-\mathrm{sel}^{-/-}$mice (Figure 4), indicating the significance of platelet P-selectin in mediating platelet accumulation during intestinal tumor growth. To determine the biological role of platelets in tumorigenesis, thrombocytopenia has been induced using antiplatelet antibodies, the thrombin inhibitor hirudin, and Nf-E2/- mice, which have almost no circulating platelets [2, 20-24]. In these models, it has been found that the deletion of platelets inhibits tumorigenesis, and plasma-purified platelets were found to significantly promote ovarian cancer cell proliferation and migration [2, 20-24], which is fully consistent with our results.

In this study, we unexpectedly found that platelet accumulation was very obvious in each and every human colorectal carcinoma or adenoma tissue sample examined. However, the present study did not elucidate a strategy to treat patients with tumors. Thus, it is highly necessary to identify a protein or compound to interfere with crosstalk between platelets and tumor cells. Interestingly, we found that P-selectin mediates the interaction between platelets and tumor cells. Therefore, it might be feasible to use the P-selectin protein to block the adhesion between 
platelets and tumor cells without severely prolonging bleeding time. Furthermore, a prospective clinical observation demonstrated that low molecular weight heparin prolonged the survival of patients with cancer in addition to exerting antithrombotic effects [25]. A recent clinical investigation also showed that post-diagnosis aspirin use increased overall colorectal cancer survival, particularly for patients with positive PTGS2 (COX-2) expression and mutated PIK3CA tumors [26]. However, high-dose heparin or aspirin may lead to bleeding, and low-dose drugs do not reduce incidence and mortality due to cancer. These data warrant further consideration, given that P-selectin-mediated platelet recruitment may be a common mechanism underlying intestinal tumorigenesis, and our experimental success in inhibiting intestinal tumor growth and tumor-induced angiogenesis with recombinant soluble P-selectin suggests its therapeutic value to treat intestinal tumors.

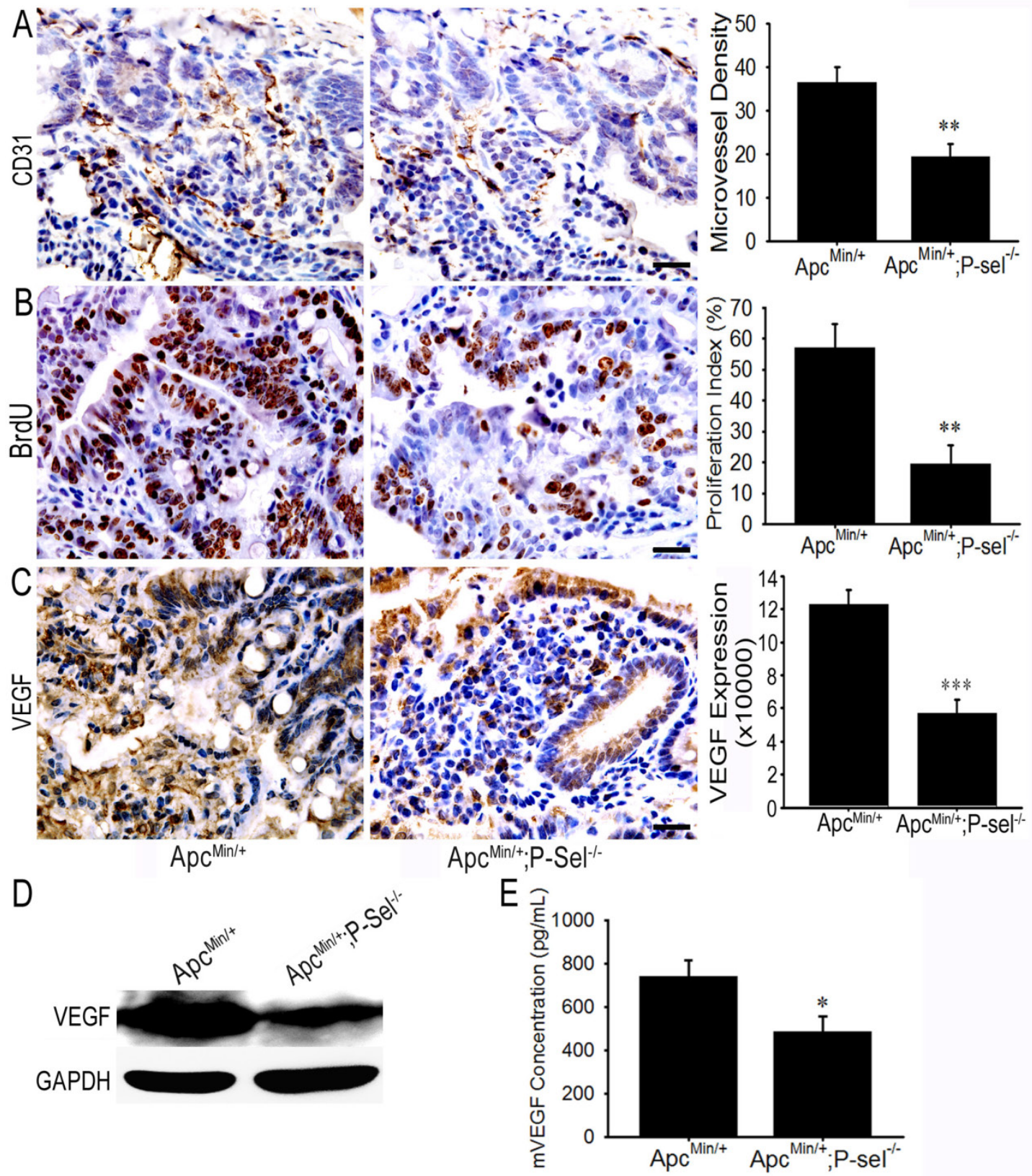

Figure 5. Adherent platelets accelerate angiogenesis via the secretion of VEGF. (A) There were fewer microvessles in the adenomas of ApcMin/+;P-sel-/mice than ApcMin/+ mice. (B) IHC staining of intestinal tumors for VEGF. VEGF expression was higher in the tumors of ApcMin/+ mice than those in ApcMin/+;P-sel-/- mice. (C) The concentration of VEGF in small intestinal adenomas. Small intestinal adenomas from ApcMin/+ and ApcMin/+;P-sel-/- mice were lysed, and the amounts of VEGF were detected by ELISA. *, $p<0.05 ; * *, p<0.01 ; * * *, p<0.001$. Scale bars $=50 \mu \mathrm{m}$. 


\section{Supplementary Material}

Figure S1. http://www.ijbs.com/v11p0679s1.pdf

\section{Acknowledgements}

The current study was supported by grants from the Doctoral Fund of the Ministry of Education of China (No. 20134423110001 to Yajie Zhang), the Science and Technology Program of Guangzhou (No. 2014Y2-00171 to Yajie Zhang), a Guangzhou Municipal Education Department Innovation team grant (No. $13 \mathrm{C06}$ to Yajie Zhang), the National Science Foundation of China (31271455 and 31471290 to Lijing Wang and 81472825 to Bo Wei), and the Science and Technology Planning Project of Gangdong Province (2013B021800082 to Cuiling Qi).

\section{Competing Interests}

The authors have declared that no competing interest exists.

\section{References}

1. Levin J, Conley CL. Thrombocytosis associated with malignant disease. Arch Intern Med. 1964;114:497-500.

2. Stone RL, Nick AM, McNeish IA, Balkwill F, Han HD, Bottsford-Miller J, et al. Paraneoplastic Thrombocytosis in Ovarian Cancer. N Engl J Med. 2012;366:610-8.

3. Borsig L. The role of platelet activation in tumor metastasis. Expert Rev Anticancer Ther. 2008;8:1247-55

4. Gimbrone MA Jr, Leapman SB, Cotran RS, Folkman J. Tumor dormancy in vivo by prevention of neovascularization. J Exp Med. 1972;136:261-76.

5. Hara Y, Steiner M, Baldini MG. Platelets as a source of growth-promoting factor(s) for tumor cells. Cancer Res. 1980;40:1212-16.

6. Pinedo HM, Verheul HM, D'Amato RJ, Folkman J. Involvement of platelets in tumour angiogenesis? Lancet. 1998;352:1775-7.

7. Ho-Tin-Noe B, Goerge T, Wagner DD. Platelets: guardians of tumor vasculature. Cancer Res. 2009;69:5623-6.

8. Furie B, Furie BC. The molecular basis of platelet and endothelial cell interaction with neutrophils and monocytes: role of P-selectin and the P-selectin ligand, PSGL-1. Thromb Haemost. 1995;74:224-7.

9. Varki NM, Varki A. Heparin inhibition of selectin-mediated interactions during the hematogenous phase of carcinoma metastasis: rationale for clinical studies in humans. Semin Thromb Hemost. 2002;28:53-66.

10. Kim YJ, Borsig L, Varki NM, Varki A. P-selectin deficiency attenuates tumor growth and metastasis. Proc Natl Acad Sci U S A. 1998;95:9325-30.

11. Nash GF, Turner LF, Scully MF, Kakkar AK. Platelets and cancer. Lancer Oncol. 2002;3:425-430.

12. Smyth SS, McEver RP, Weyrich AS, Morrell CN, Hoffman MR, Arepally GM, et al. Platelet functions beyond hemostasis. J Thromb Haemost. 2009;7:1759-66.

13. Su LK, Kinzler KW, Vogelstein B, Preisinger AC, Moser AR, Luongo C, et al. Multiple intestinal neoplasia caused by a mutation in the murine homolog of the APC gene. Science. 1992;256:668-70.

14. Qi C, Zhou Q, Li B, Yang Y, Cao L, Ye Y, Li J, et al. Glipizide, an antidiabetic drug, suppresses tumor growth and metastasis by inhibiting angiogenesis. Oncotarget. 2014;5:9966-79.

15. Raufman JP, Shant J, Xie G, Cheng K, Gao XM, Shiu B, et al. Muscarinic receptor subtype- 3 gene ablation and scopolamine butylbromide treatment attenuate small intestinal neoplasia in Apcmin/+ mice. Carcinogenesis. 2011;32:1396-402.

16. Qi CL, Wei B, Ye J, Yang Y, Li B, Zhang QQ, et al. P-selectin-mediated platelet adhesion promotes the metastasis of murine melanoma cells. PLoS One. 2014; 9:e91320.

17. Gasic GJ, Gasic TB, Galanti N, Johnson T, Murphy S. Platelet-tumor-cell interactions in mice. The role of platelets in the spread of malignant disease. Int J Cancer. 1973;11:704-18.

18. Boivin GP, Washington K, Yang K, Ward JM, Pretlow TP, Russell R, et al. Pathology of mouse models of intestinal cancer: consensus report and recommendations. Gastroenterology. 2003;124:762-77.

19. Dvorak HF. Tumors: wounds that do not heal. Similarities between tumor stroma generation and wound healing. N Engl J Med. 1986;315:1650-9.
20. Camerer E, Qazi AA, Duong DN, Cornelissen I, Advincula R, Coughlin SR. Platelets, protease-activated receptors, and fibrinogen in hematogenous metastasis. Blood. 2004;104:397-401.

21. Karpatkin S, Pearlstein E, Ambrogio C, Coller BS. Role of adhesive proteins in platelet tumor interaction in vitro and metastasis formation in vivo. J Clin Invest. 1988; 81:1012-9.

22. Kato Y, Fujita N, Yano H, Tsuruo T. Suppression of experimental lung colonization of mouse colon adenocarcinoma 26 in vivo by an anti-idiotype monoclonal antibody recognizing a platelet surface molecule. Cancer Res. 1997; 57:3040-5.

23. Trikha M, Zhou Z, Timar J, Raso E, Kennel M, Emmell E, Nakada MT. Multiple roles for platelet GPIIb/IIIa and alphavbeta3 integrins in tumor growth, angiogenesis, and metastasis. Cancer Res. 2002;62:2824-33.

24. Amirkhosravi A, Mousa SA, Amaya M, Blaydes S, Desai H, Meyer T, Francis JL. Inhibition of tumor cell-induced platelet aggregation and lung metastasis by the oral GpIIb/IIIa antagonist XV454. Thromb Haemost. 2003;90:549-54.

25. Ho-Tin-Noé B, Goerge T, Wagner DD. Platelets: guardians of tumor vasculature. Cancer Res. 2009;69:5623-6.

26. Goh HH, Leong WQ, Chew MH, Pan YS, Tony LK, Chew L, et al. Post-operative Aspirin Use and Colorectal Cancer-specific Survival in Patients with Stage I-III Colorectal Cancer. Anticancer Res. 2014;34:7407-14. 